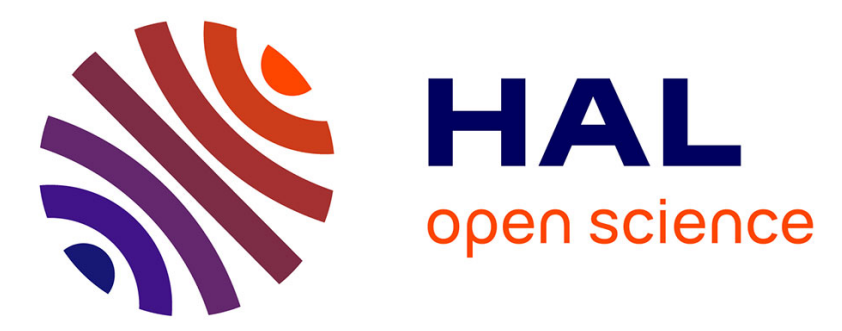

\title{
Electron tomography on latex particles suspended in water using Environmental Scanning Electron Microscopy
}

\author{
X. Jiao, Lucian Roiban, G. Foray, Karine Masenelli-Varlot
}

\section{To cite this version:}

X. Jiao, Lucian Roiban, G. Foray, Karine Masenelli-Varlot. Electron tomography on latex particles suspended in water using Environmental Scanning Electron Microscopy. Micron, 2019, 117, pp.60-67. 10.1016/j.micron.2018.11.007 . hal-02156643

\section{HAL Id: hal-02156643 \\ https://hal.science/hal-02156643}

Submitted on 14 Jun 2019

HAL is a multi-disciplinary open access archive for the deposit and dissemination of scientific research documents, whether they are published or not. The documents may come from teaching and research institutions in France or abroad, or from public or private research centers.
L'archive ouverte pluridisciplinaire HAL, est destinée au dépôt et à la diffusion de documents scientifiques de niveau recherche, publiés ou non, émanant des établissements d'enseignement et de recherche français ou étrangers, des laboratoires publics ou privés. 


\title{
Electron tomography on latex particles suspended in water using Environmental Scanning Electron Microscopy
}

\author{
X. Jiao ${ }^{1}$, L. Roiban ${ }^{1 *}$, G. Foray ${ }^{1}$ and K. Masenelli-Varlot ${ }^{1 *}$ \\ 1. Univ Lyon, INSA-Lyon, UCBL, MATEIS, CNRS UMR 5510, F-69621 Villeurbanne, France \\ * corresponding authors : Lucian.Roiban@insa-lyon.fr ; Karine.Masenelli-Varlot@insa-lyon.fr
}

\begin{abstract}
Tilted image series are acquired on liquid suspensions of latex particles supported by a carbon membrane. To do so, a home-made stage fitting in an Environmental Scanning Electron Microscope (ESEM) is optimized in order to minimize cold losses and therefore improve both sample stability and spatial resolution in the images. As latex particles are electron-sensitive materials, sample degradation and stability are taken into account in the choice of the parameters for tilted image series acquisition. Despite the limited number of images, conventional algorithms are used to reconstruct the volume and study the three-dimensional arrangement of latex particles. The lattice types are in good agreement with what can be observed on frozen suspensions, although the domain sizes are smaller. Several quantitative parameters such as the latex particle size and the distance to the first neighbors are extracted from the 3D model. Finally, the spatial resolution in the tomograms is estimated using the Crowther's criterion and Fourier Shell Correlation analysis. It is also measured directly by analyzing a suspension containing latex particles and a surfactant of interest for composite formulation.
\end{abstract}

Keywords: ESEM, STEM, liquid, electron tomography, polymer

\section{Introduction}

The use of liquid phase electron microscopy has largely increased during the last decade with the commercialization of liquid cell sample holders fitting in Transmission Electron Microscopes (TEMs) operating under vacuum. Indeed, when compared with cryo-TEM for instance, it has become possible to 
perform in situ heating or electrochemical experiments (Ross 2015). Several kinds of materials can be studied, but in complex samples, two dimensional images could not provide some accurate threedimensional (3D) information (Tan et al. 2011). Moreover, knowing that several kinds of materials contain water or are dispersed in water during their process, it is important to characterize 3D arrangement in the liquid. This may open routes to optimize the fabrication process, to check the quality of the microstructure built and last but not least taylor the final material's functional properties. This is also true when studying the movement of nanoparticles in liquid (Proetto et al., 2014) (Verch et al., 2015). Electron tomography is in this case a key technique to obtain and quantify a material in 3D.

The presence and geometry of the silicon nitride windows in liquid cell sample holders hinder the acquisition of tilted image series as the tilting capabilities are extremely limited. An alternative could come from the use of graphene as thin membrane (Yuk et al. 2012) (Park et al., 2015). Environmental Scanning Electron Microscopy (ESEM) is also a perfectly suited technique for the analysis of waterbased materials (Stokes, 2008). Indeed, liquid water can be preserved inside the microscope chamber thanks to differential pumping allowing the presence of a gaseous environment around the sample, while the microscope column is kept under high vacuum (Donald, 2003). Moreover, water vapor is an efficient gas amplifier for secondary electrons (Fletcher et al., 1997). The observation of heterogeneous liquids (liquid emulsions or liquid containing solid nano-objects) or hydrated samples with a resolution of a few or a few tens nanometers is possible when working in transmission in the ESEM (Bogner et al., 2005) (Peckys et al., 2013).

Interestingly, we have previously shown that electron tomography can be carried out in the ESEM chamber with this STEM-in-SEM mode on dry samples (Jornsanoh et al., 2011) or during in situ condensation experiments (Masenelli-Varlot et al., 2014). The aim of this paper is to demonstrate the feasibility of electron tomography on aqueous suspensions in their native state. This implies to go one step further in as the liquid state should not only be preserved when introduced into the microscope chamber, but it should also be stable all along the acquisition of tilted image series. Latex particles dispersed in water are well-suited materials to investigate the stability of the water film. Indeed, latex particles are spherical when dispersed into water but form homogeneous films upon drying (Chevalier et al., 1992). This irreversible transformation is a well-suited probe for the stability of the water film, as it will not be possible to come back to the native state once dried. The drawback is that polymers give low 
contrasts in electron microscopy and are highly electron sensitive. Water hydrolysis under electron irradiation (Schneider et al., 2014), depending on several experimental parameters (Abellan et al., 2014) needs also to be taken into account, as the formation of hydrated electrons and free radicals in water may diffuse at the water-polymer interface and promote polymer degradation.

\section{Materials \& Methods}

SBA-PMMA is a copolymer derived from styrene and metacrylic acid esters in aqueous solution. The SBA particle diameter is monodispersed and centered on $200 \mathrm{~nm}$. The glass transition temperature is around $4^{\circ} \mathrm{C}$. This latex is a model molecule provide by BASF R\&D lab, designed to understand cement latex interaction. The SBA particles already include a 3\% PMMA shell, playing the role of steric surfactant. Another surfactant (XPCAS 803 from PCAS) may be used in addition to PMMA. It has a high molecular weight and combines steric and ionic functions and is currently used to form composites. The dilution of the final suspension is adjusted prior to the experiment, so that either individual or densely packed SBA-PMMA particles can be observed.

Observations are carried out in an ESEM XL-30 FEG from FEI. The acceleration voltage is set to 30 $\mathrm{kV}$. To minimize the skirt effect and obtain the best possible resolution, a gaseous annular detector equipped with a $9 \mathrm{~mm}$ long cone is placed below the objective lens and the working distance is set to 10 to $11 \mathrm{~mm}$. A solid-state electron detector placed below the sample collects the scattered signal. The sample-to-detector distance is $10 \mathrm{~mm}$, so that the detector collects electrons scattered between 250 to $700 \mathrm{mrad}$. The probe current is measured to be equal to $240 \mathrm{nA}$ for a probe size of $8 \mathrm{~nm}$ (probe size 3 ). $1290 * 968$ images are acquired with a dwell time of $3.36 \mathrm{~ms} / \mathrm{line}$. Such value of the dwell time ensures a good enough signal-to-noise ratio. At the same time, no significant morphological change is detected after the tilt series acquisition using this acquisition time.

The STEM sample holder is placed on a tomographic stage described elsewhere (Masenelli-Varlot et al., 2014). Briefly, this stage is composed of 5 main parts: a $360^{\circ}$-rotating stage, two translating piezoelectric systems to place the sample to the eucentric position, a Peltier stage equipped with a thermocouple and connected to the FEI thermoelectric stage controller, and a tweezer-like sample holder. In this set-up, where the liquid is not encapsulated into any closed cell, it is crucial to stay at the liquid-vapor equilibrium to avoid any major change in the water film thickness (that is to say no 
evaporation or condensation). Preliminary experiments using latex suspensions (not shown here) revealed severe water evaporation on the sample, although the chosen Peltier stage temperature and gas pressure values were supposed to correspond to the liquid-vapor equilibrium. Actually, the sample temperature was higher than expected due to thermal losses from the Peltier stage to the sample position. Therefore, additional thermal insulating layers have been placed on the Peltier stage and a new sample holder has been designed, see Figure 1a. Temperature measurements have been carried out using an external thermocouple placed very close to the sample position. In order to approach operating conditions, a droplet of pure water has been deposited onto a TEM copper grid covered with a holey carbon membrane. Two sets of temperature $\left(\mathrm{T}_{1}, \mathrm{~T}_{2}\right)$, measured independently from each other at different water vapor pressures, are represented in Figure 1b. Interestingly, the temperature is reproducible from one experiment to the other. Yet, for one water vapor pressure, the temperature on the sample is systematically higher by $1^{\circ}$ to $2{ }^{\circ} \mathrm{C}$ than the liquid-vapor equilibrium temperature. Such shift could partially be attributed to the thermocouple uncertainty. Nevertheless, as the shift is reproducible, it can be used as a calibration curve instead of the water dew curve. During the experiments, the target sample temperature and water vapor pressure values are chosen according to this calibration curve.
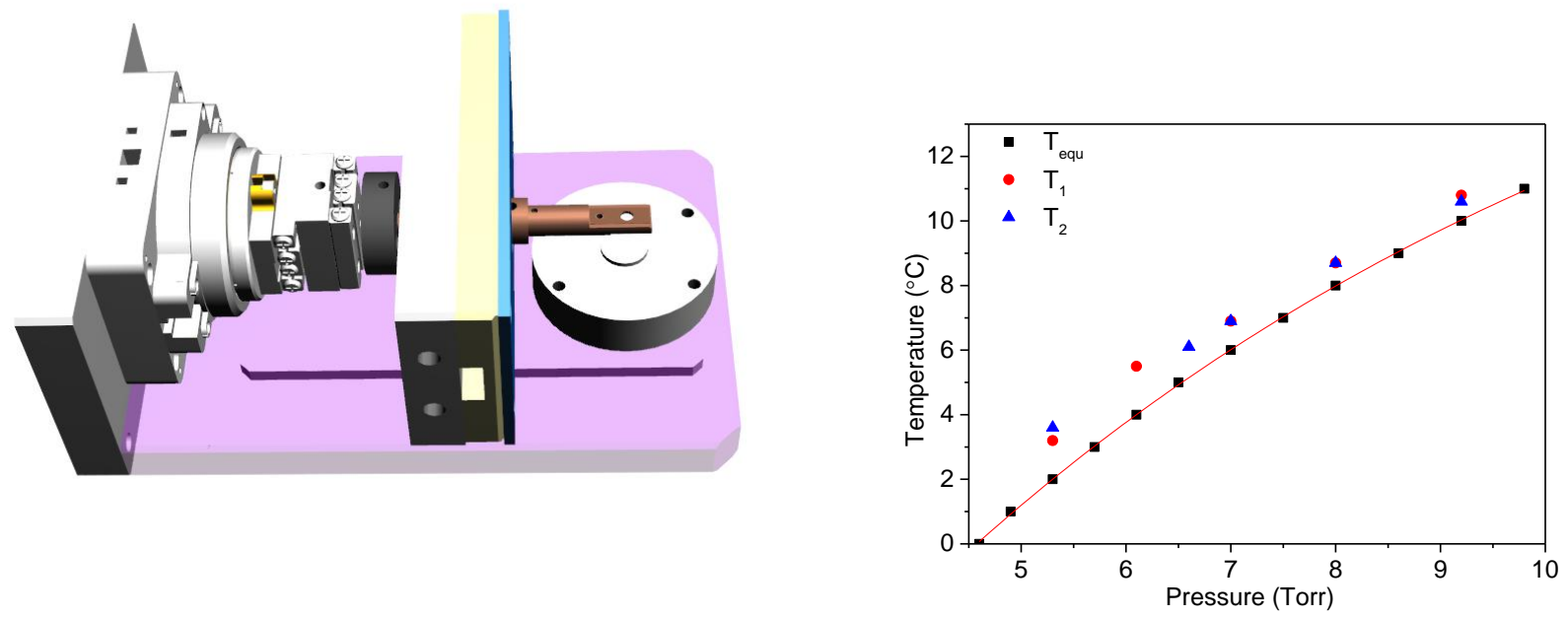

b)

a)

Figure 1: design of the tomographic stage and temperature measurements. a) side view showing the insulating materials on the Peltier element (in blue) and a thick sample holder. b) temperature measured as a function of the water pressure, on the sample holder covered with a droplet of pure water. Two sets of temperature $\left(T_{1}, T_{2}\right)$, measured independently, are represented. Whatever the pressure, the temperature is systematically higher than the temperature of water at the liquid-vapor equilibrium state $\left(\mathrm{T}_{\text {equ }}\right)$.

A $1 \mu 1$ droplet of suspension is deposited with an Eppendorf micropipette onto a 300-mesh TEM copper 
grid covered with a holey carbon film and decorated with gold nanoparticles (diameter $40 \mathrm{~nm}$ ) synthesized according to a procedure described elsewhere (Ziegler \& Eychmüller, 2011). The temperature of the Peltier stage is set at $2^{\circ} \mathrm{C}$, which leads to a sample temperature equal to around $3.5^{\circ} \mathrm{C}$. The parameters of the purge sequences ( 8 cycles between 6.5 and 9 Torr) are chosen to avoid any dehydration of the suspension. Moreover, a few droplets of water are first deposited on the stage outside the Peltier stage, to saturate the sample environment with water vapor during the purge sequence. The water vapor pressure is then fixed to around 5.5 Torr. It is slowly decreased to evaporate water from the sample, until it becomes electron transparent, then the pressure is set again to the equilibrium pressure.

Tilted image series are acquired with the STEM detector, usually from $-60^{\circ}$ to $+60^{\circ}$ with a tilt step of $5^{\circ}$. The imaging conditions are also optimized to reduce irradiation damage. For example, for each tilt angle, the focus, contrast and brightness in the images are not adjusted directly on the region of interest but in a region located nearby (Roiban et al., 2016), on the tilt axis. With a pixel size of $7.5 \mathrm{~nm}$, one pixel receives around 3900 electrons during the acquisition of one tilted image. After the tilt series acquisition, an accurate alignment of the projections is performed using IMOD (Kremer et al., 1996), using gold nanoparticles as fiducial markers. The volume reconstruction is done using the TomoJ plugin (Messaoudi et al., 2007) of ImageJ software, with the ART algorithm (15 iterations). Segmentation is carried out combining Trainable Waka Segmentation, a plug-in in Fiji, and 3D Slicer (http://www.slicer.org/) (Fedorov et al., 2012), further used for advanced segmentation. Fourier Shell Correlation analysis is used to estimate the spatial resolution in the reconstructed volume. The software available free of charge from the Image Science website (www.imagescience.de) is used with two reconstructed volumes calculated with odd and even images.

Cryo-SEM experiments are performed on a FEI Quanta 250 FEG scanning electron microscope. A droplet of suspension is frozen into liquid nitrogen and transferred into the microscope chamber using a cryo transfer Alto 2500 from Gatan. Secondary electron images are acquired under high vacuum using an acceleration voltage of $2 \mathrm{kV}$.

Monte Carlo simulations are performed using the free software Hurricane from SAMx (http://www.samx.com/index.html.fr). The geometry used is similar to the one published elsewhere (Xiao et al., 2018). Briefly, a water film of thickness ranging between $200 \mathrm{~nm}$ and $1 \mu \mathrm{m}$ is defined. Its 
lateral dimensions is chosen to be equal to at least $5 \mu \mathrm{m}$, so that a negligible amount of electron escape through these sides. A spherical particle of pure SBA (chemical composition $\mathrm{C}_{7.45} \mathrm{H}_{10.2} \mathrm{O}_{1.1}$; density 1.07 g. $\mathrm{cm}^{-3}$; radius $100 \mathrm{~nm}$ ), is placed in the middle of the water film. The PMMA shell is not included into the model. An electron beam (diameter $1 \mathrm{~nm}$; energy $30 \mathrm{keV}$ ) is placed on the top of the SBA particle. The trajectories of 1,000,000 electrons are calculated. The number of collected electrons correspond to the number of incident electrons that pass through the sample and that are scattered with angles ranging between 250 and 700 mrad.

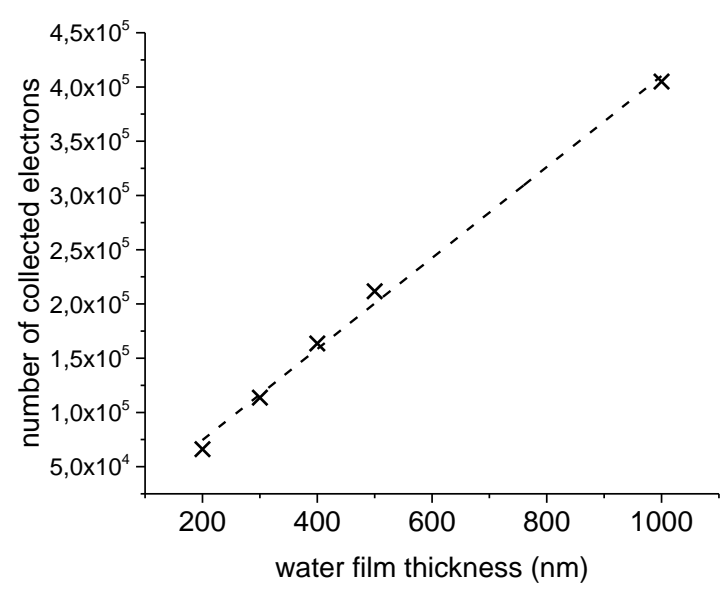

a)

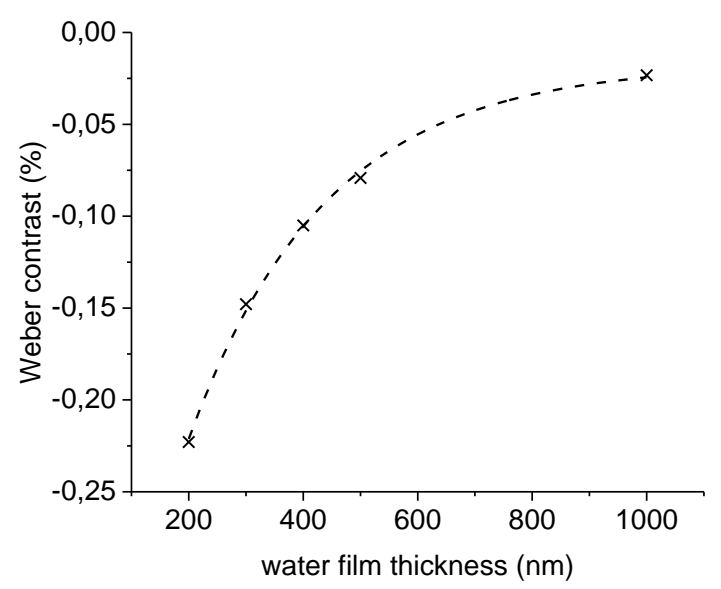

b)

Figure 2: Monte Carlo simulation results on a SBA sphere suspended in water. a) the number of collected electrons increases almost linearly with the water film thickness, in the range $200 \mathrm{~nm}-1 \mu \mathrm{m}$. b) the Weber contrast decreases exponentially with the water film thickness but remains negative.

\section{Results \& Discussion}

Figure 1a displays the number of collected electrons computed using Monte Carlo simulations in the case of a SBA particle suspended in water of variable thickness. For water thicknesses ranging between $200 \mathrm{~nm}$ and $1 \mu \mathrm{m}$, the number of collected electrons increases almost linearly. It is also worthy to compute the contrast between a SBA sphere and the surrounding water film to ensure that no contrast inversion will occur. Weber contrast can be defined as $W=\frac{n_{S B A}-n_{H 2 O}}{n_{H 20}}$. For each point, $n_{S B A}$ represents the number of collected electrons from Figure 1a. $n_{H 2 O}$ corresponds to the number of collected electrons outside the SBA particle, i.e. on the water background. The evolution of the Weber contrast with the water film thickness is given in Figure 1b. The contrast is negative, which means that the SBA particles 
are always darker than water. When increasing the water film thickness, the Weber contrast decreases almost exponentially. No contrast inversion occurs in this range.

Figure 3 shows STEM images acquired at different tilt angles on a sample composed of a diluted suspension of SBA-PMMA latex particles deposited onto a holey carbon membrane. The images are extracted from a tilted image series at $+20^{\circ}, 0^{\circ}$ and $-20^{\circ}$. A thin layer of water covers the smallest holes in the membrane, whereas the bigger holes $(1 \mu \mathrm{m}$ or more) are not covered. The latex particles appear darker than the surrounding water film, as expected from the simulations performed on pure SBA particles (see Figure 1). When compared to Monte Carlo simulations published elsewhere (Xiao et al., 2018), the grey value profile along one SBA-PMMA latex particle corresponds to a relatively thick water layer (in the order of magnitude of $400 \mathrm{~nm}$ according to the simulations published). No large change such as water evaporation can be observed apparently, apart from a thickness variation of the water film covering the holes in the carbon membrane. Such change can be observed for instance on the hole located at the center of the images that is partially covered. It is attributed to instabilities of the water film at low thickness due to irradiation-induced hydrolysis (Schneider et al., 2014). Nevertheless, the latex particles do not seem to be significantly affected and it is noteworthy that they do not move significantly during the tilted image series acquisition, even when the sample is tilted at $\pm 60^{\circ}$. This could first be explained by their large size and by the low sample temperature, two key parameters for Brownian motion (Jan \& Choi, 2004) (Chon et al., 2005). The latex particles are also probably immobilized between the carbon membrane and the water film top surface through a joint effect of Van der Waals forces and surface tension.

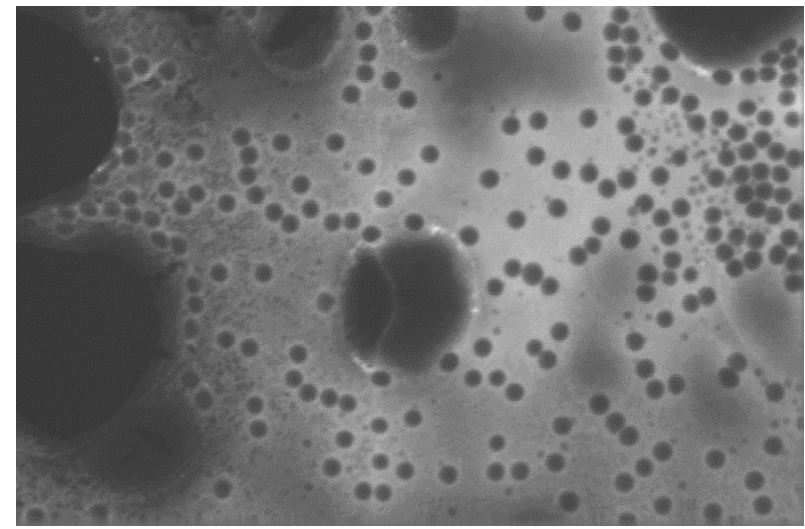

a)

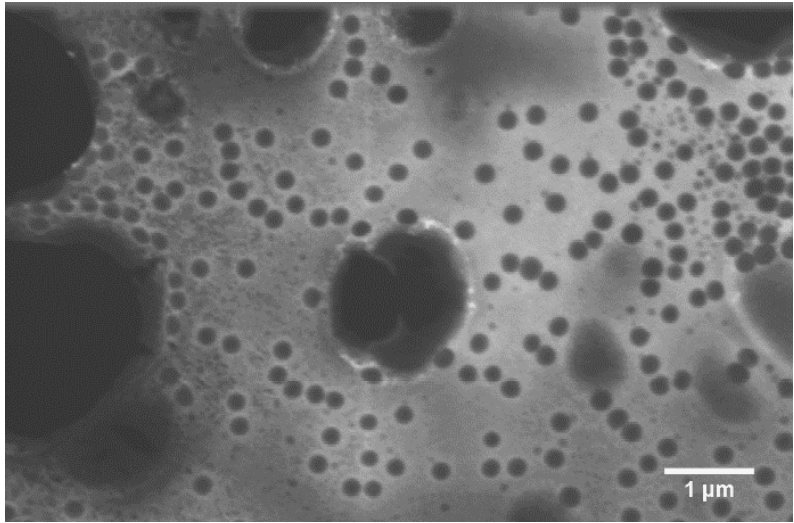

b) 


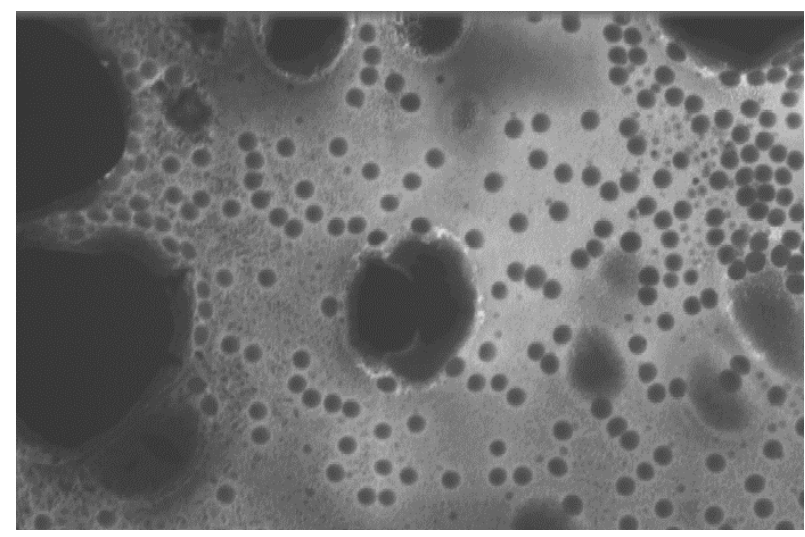

c)
Figure 3: images extracted from an aligned tilt series. Images a), b) and c) correspond to tilts of $+20^{\circ}, 0^{\circ}$ and $-20^{\circ}$ respectively. The latex particles (darker gray dots) are fixed on the holey carbon membranes.

On a more concentrated suspension, it becomes more difficult to identify individual latex particles when they form multiple layers on the carbon membrane. Figure 4a gives a typical image, with the formation of dense lattices on the carbon membrane. In this case, the latex spheres are still darker than water, which suggests a rather thick water film thickness. Similarly to what is observed with the diluted suspension, the hole in the carbon membrane is too big to ensure a good stability of the water film. Consequently, the interfaces between latex spheres are not visible anymore and a uniform film is formed (Chevalier et al., 1992).

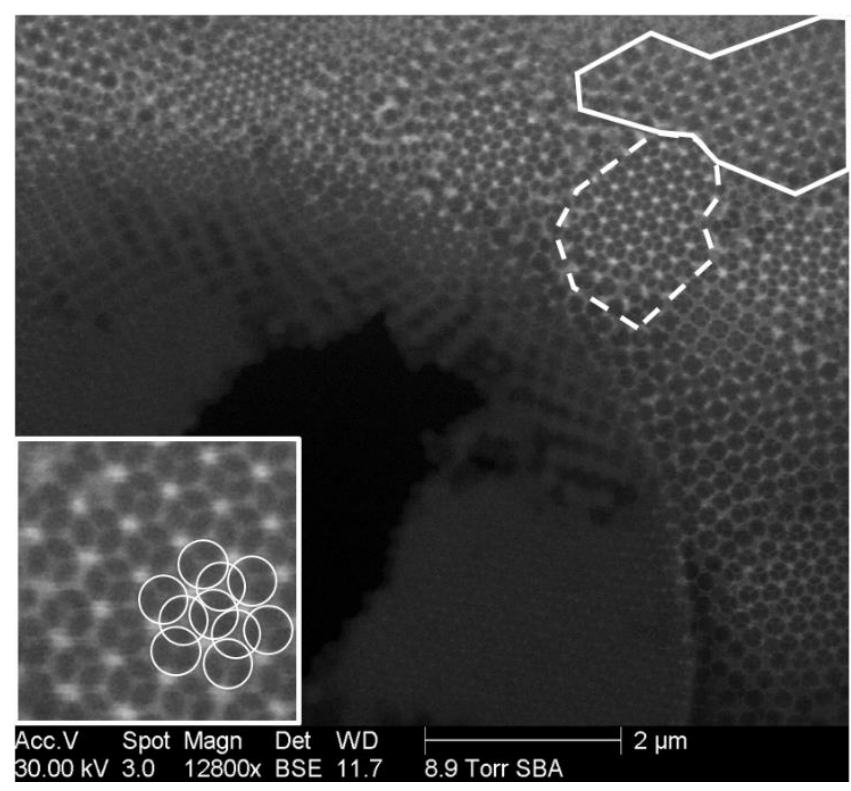

a)

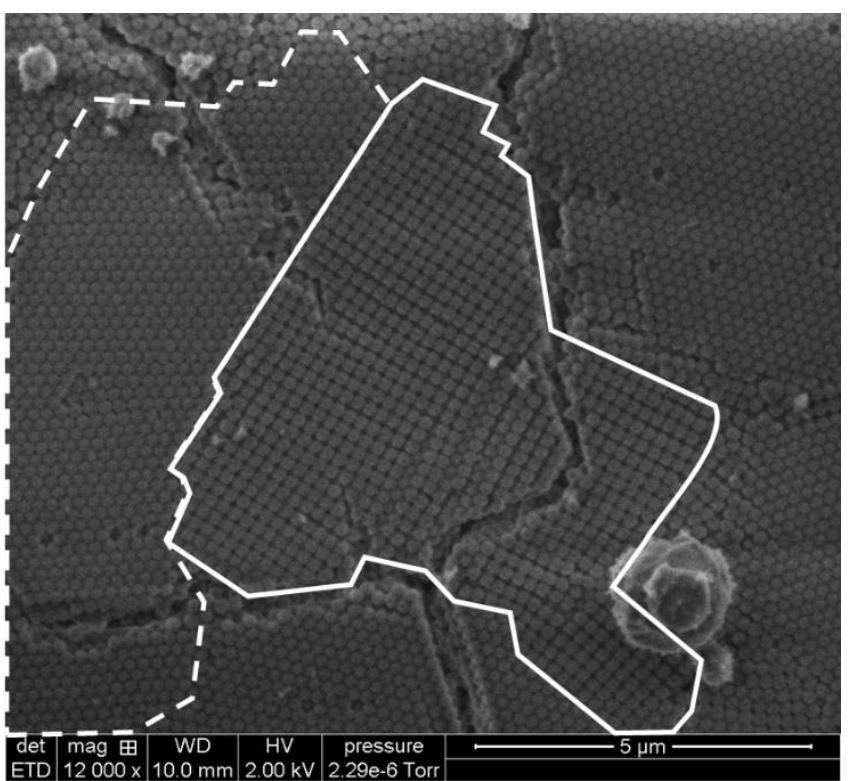

b)

Figure 4: Comparison between images of a concentrated suspension of latex particles, acquired in the liquid and in the frozen states. The bold lines enhance the moncrystaline domains in cubic assembly and the dash lines enhance domains in hexagonal 
assembly. The inset is a zoom of the domain exhibitin a hexagonal arrangement. The circles correspond to the latex spheres. a) STEM image acquired on the liquid at $0^{\circ}$. b) secondary electron image acquired in cryo-SEM.

On the carbon membrane, either mono- or multilayers of latex particles can be distinguished.

Monocrystalline domains are clearly visible, with either cubic or hexagonal lattices (see bold or dashed lines in Figure 4a, respectively). The existence of both lattice types is representative of what occurs at bulk. Indeed, Figure $4 \mathrm{~b}$ displays a typical image obtained using cryo-SEM on a frozen droplet of the same suspension. The secondary electron image gives insight on the droplet surface topography. Clearly, cubic and hexagonal lattices are formed within the droplet. Interestingly, the "monocrystal" assembly sizes are significantly larger in cryo-SEM than using STEM-in-SEM on the liquid. The difference could be attributed to dissimilar experimental conditions: the onset of the ordering process is known to occur when the thickness of the water layer becomes equal or smaller than the latex particle diameter (Denkov et al., 1992). In liquid, the presence of a meniscus most probably creates a higher number of nucleation sites when compared with cryo-SEM experiments. Such meniscus is of interest as it represents the reality of composite elaboration, with multiscale pores and fillers. Moreover, the evaporation rate has been found to play a major role onto the domain size, with very large and wellordered domains formed at low evaporation rate (Denkov et al., 1992). Therefore, the domain size cannot be accurately measured using STEM-in-SEM on liquids as the experimental conditions can up to now not accurately reproduce the usage conditions. Nevertheless, it may possible to study in detail the ordering process as a function of the suspension composition.
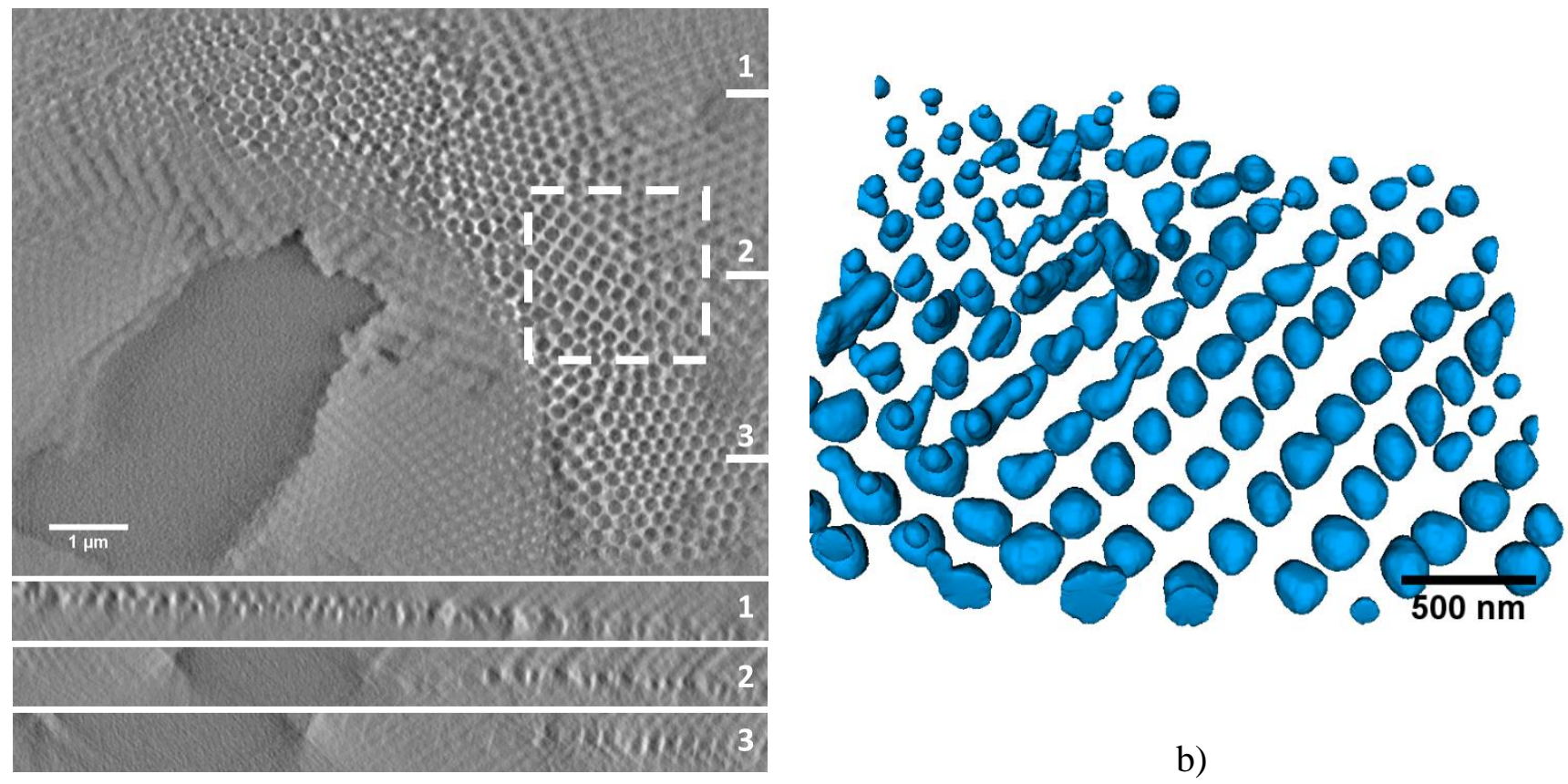

b) 
a)

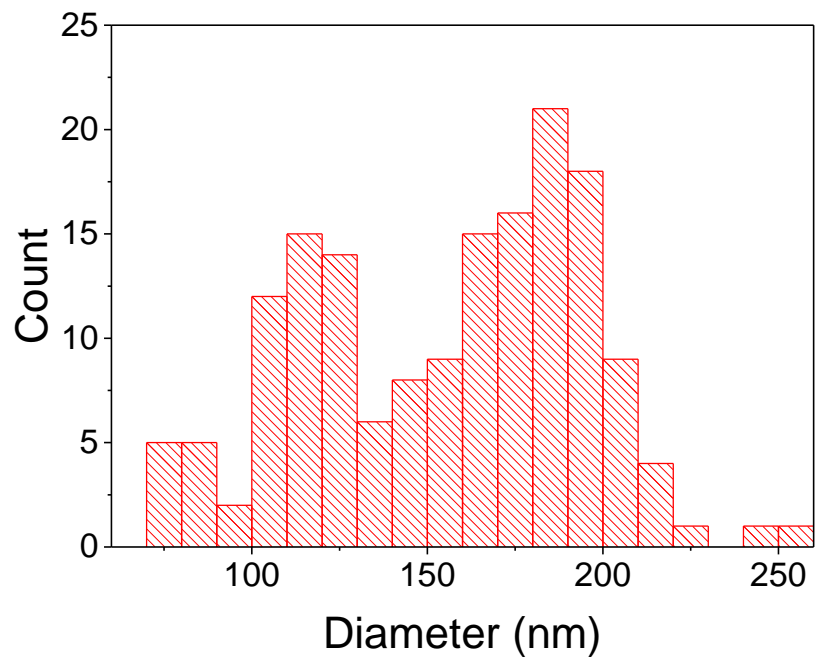

c)

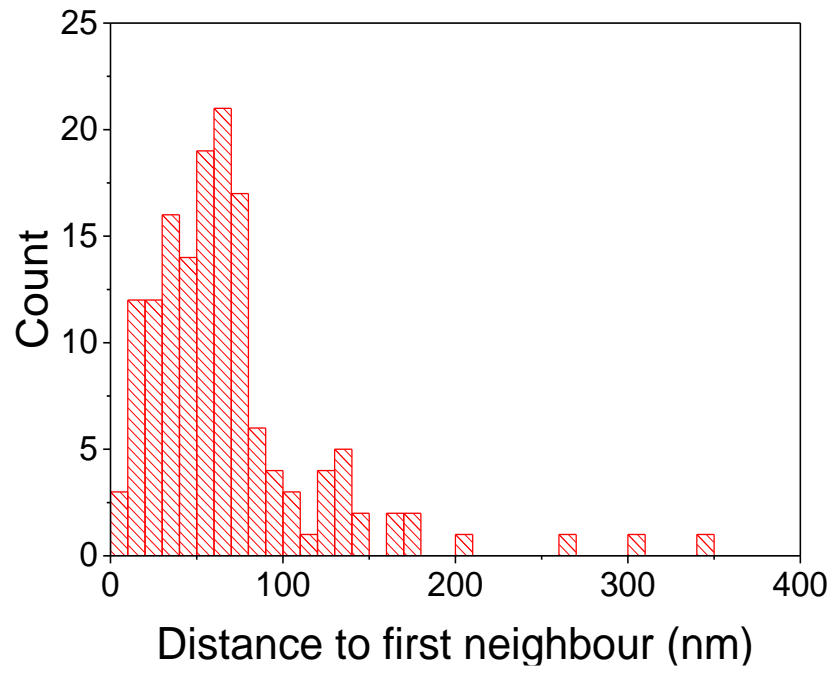

d)

Figure 5: 3D ordering of SBA-PMMA latex particles in liquid water. a) orthogonal slices paralel to the XY (top) and XZ (bttom 1,2 and 3) planes extracted fom the reconstructed volume of size $X * Y * Z=9488 * 7163 * 3000 \mathrm{~nm}^{3}$. The short white lines indicate the position of the slices. b) 3D model of the region indicated by the white square in a) (model volume: $\mathrm{X}^{*} \mathrm{Y} * \mathrm{Z}=$ $\left.2220 * 2430 * 1560 \mathrm{~nm}^{3}\right)$; c) particle size distribution measured from the 3D model and d) distance to the first neighbour, measured from the 3D model.

A slice parallel to the XY plane extracted from the reconstructed volume is given in Figure 5a. The use of a large tilt step and the presence of a missing wedge of $60^{\circ}$ induces fanning and elongation in the $\mathrm{z}$ direction (Midgley \& Weyland, 2003). Despite such artifacts, it is possible to distinguish individual latex particles forming mono- or bilayers. The 3D model obtained after segmentation of the region indicated by a white square in the reconstructed volume is displayed in Figure 5b. This region corresponds to the transition between a mono- and a bilayer. The latex particles found after segmentation are not purely spherical, as expected, because of the presence of artifacts. The diameter distribution of latex particles, measured from the 3D model, is shown in Figure 5c. The uncertainty associated to each measure corresponds to a systematic uncertainty of about $30 \mathrm{~nm}$, which corresponds to 1 dilatation of 4 pixels of $7.5 \mathrm{~nm}$ each during the segmentation process. Interestingly, the diameter distribution exhibits a maximum around $180 \mathrm{~nm}$, which is in very good agreement with measurements performed on 2D images (Perret et al., 2018). However, a second mode around $110 \mathrm{~nm}$ is present on the diameter distribution. It corresponds to poorly resolved latex particles on the second layer (upper left corner in Figure 5b), probably due the elongation factor that overlaps nanoparticles volumes. Such distortions could be reduced for instance by building a 3D model containing only spheres but in this case, it would 
be impossible to take into account the latex particle deformation upon water deformation (Chevalier et al., 1992).

In the 3D model, the latex particles form a simple cubic lattice. The distribution of the distances to the first neighbours is given in Figure 5d. The uncertainty on each measure is also estimated to be equal to $30 \mathrm{~nm}$. The distance distribution is an important parameter in the process of film formation. The mean distance to the first neighbours is around $80 \mathrm{~nm}$ and fairly corresponds to the distance between particles in one layer. A few particles are found to be in contact with each other (distance to first neighbor equal to 0 ). They are mainly placed at the interface between the mono- and the bilayer, as expected from the supposed shape of the water film meniscus. Indeed, a thin layer of water induces a contact between particles and hence coalescence, as described in the literature (Chevalier et al., 1992) (Denkov et al., 1992). Interestingly in the film formation proposed in the literature, the latex particles form a hexagonal compact lattice before coalescence. We show here that they can also form a cubic lattice, even though it is less dense than the hexagonal one.

Spatial resolution is a key issue in electron tomography. According the Crowther criterion, the resolution $\mathrm{d}$ in the reconstructed volume can be estimated as $\pi \mathrm{D} / \mathrm{N}$, where $\mathrm{D}$ is the diameter of the spherical object and $\mathrm{N}$ the number of tilted images (Crowther et al., 1970). In our case, if one consider that the object is an individual latex particle of diameter $180 \mathrm{~nm}$ - and not the water film thickness of the thickness of the particle bilayer - the spatial resolution given by the Crowther's criterion would be equal to $23 \mathrm{~nm}$. Fourier Shell Correlation (FSC) analysis has been developed to provide a more realistic estimation of the spatial resolution (Harauz \& Van Heel, 1986), as the Crowther's criterion often overestimates the spatial resolution. The FSC curve corresponding to the reconstructed volume displayed in Figure 5a is given in Supporting Information. The resolution measured with the $\sigma$-criterion is equal to $31 \mathrm{~nm}$. From a theoretical point of view, this value represents the minimum distance for which information is significantly above the noise level (Van Heel \& Schatz, 2005). Interestingly, the values provided by the Crowther's criterion and by the FSC analysis are in fair agreement and are both equivalent to the estimated uncertainty.

In order to investigate more deeply the resolution which can be obtained on aqueous suspensions of latex particles, a surfactant is added to the suspension. The sizes of the surfactant shell and the latex core have been quantitatively measured in liquid and in the dry state (Perret et al., 2018). Figure 6a and 
Figure $6 \mathrm{~b}$ display the STEM image acquired at $0^{\circ}$ extracted from a tilted image series, and orthogonal cross sections extracted from the reconstructed volume, respectively. The surfactant forms a bright shell around the latex particles, as found previously. On the contrary to the study previously published, the surfactant fraction has been adjusted to obtain a uniform layer around the latex particles and no surfactant in excess. It is noteworthy that the surfactant shells are brighter in a small rectangle at the center of Figure 6a, which is direct evidence of irradiation damage. All the shells are visible in the reconstructed volume, even in the less irradiated regions.

In order to minimize the fanning and elongation effects in the 3D model, the reconstructed volume is modeled with perfect spheres. The radius of the spheres is chosen manually in order to obtain a good fit with the grey level variations in the reconstructed volume. The 3D core-shell model obtained is represented in Figure 6c, with a surfactant shell thickness of around $25 \mathrm{~nm}$. The particles arrangement in two layers is similar to that observed on the suspension without surfactant. However, the locally less dense suspension does not exhibit dense packing but rather small clusters/embryos. Interestingly, it is possible to visualize how the surfactant shell behaves when the latex particle get in close contact. Indeed, some particles are still separated by both surfactant shells (see on the left part of Figure 6c) whereas other ones are separated by one shell only (see at the center of Figure $6 c$ ). This clearly indicates an interpenetration of the surfactant shells, as proposed in the literature (Du Chesne et al., 1997) (Tzitzinou et al., 1999). 


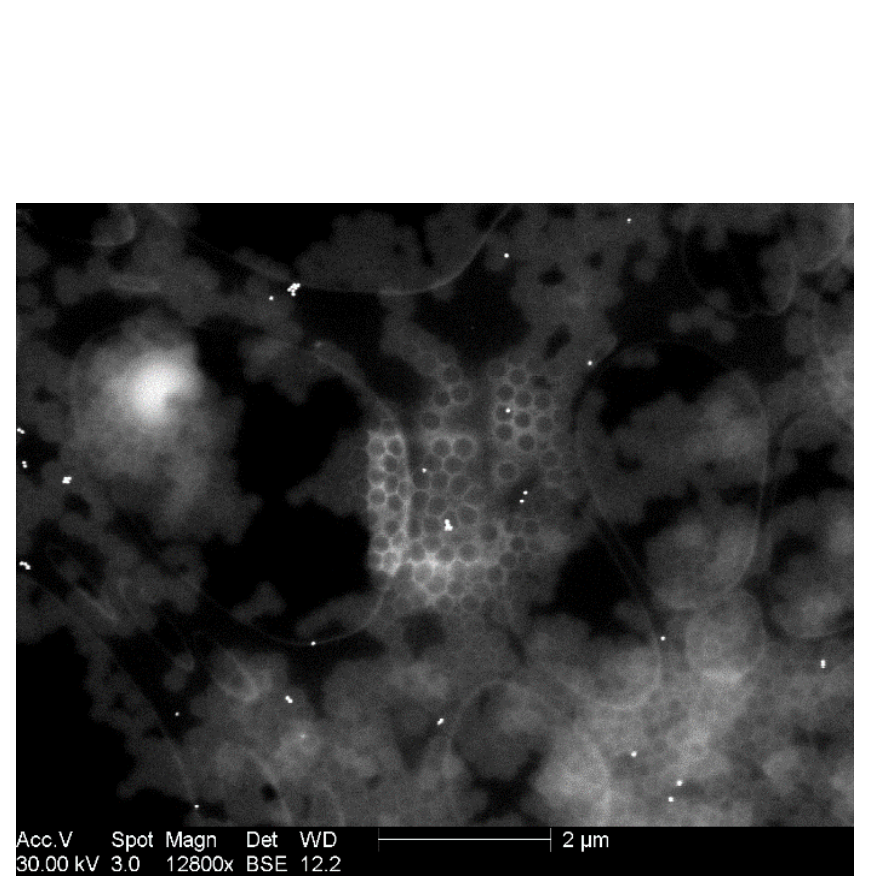

a)

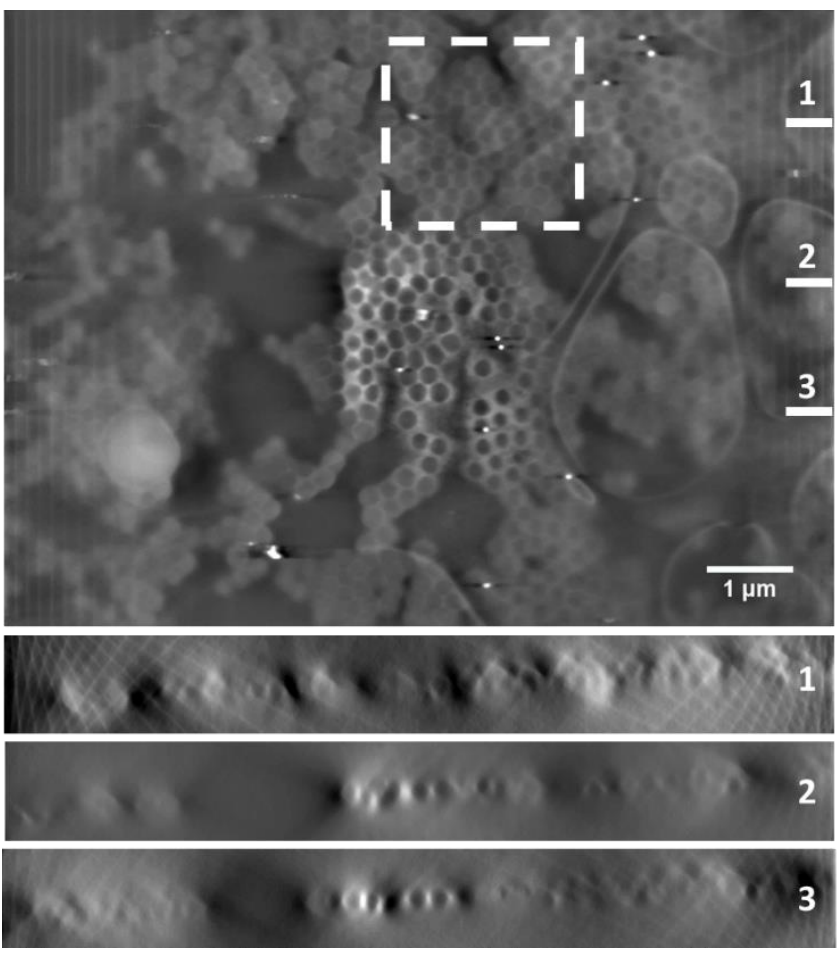

b)

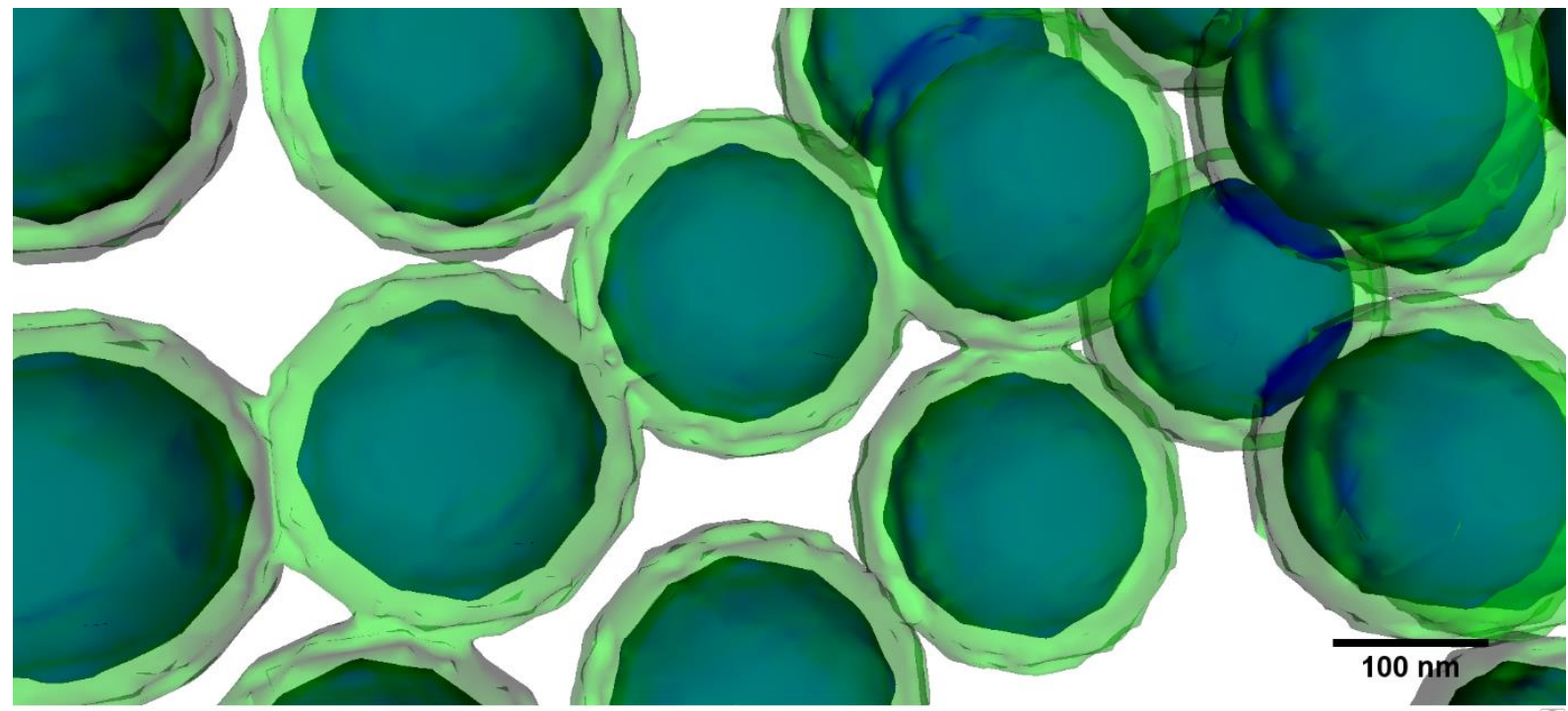

c)

Figure 6: 3D ordering of SBA-PMMA latex particles in liquid water in presence of a surfactant. a) STEM image acquired at $0^{\circ}$. The white dots are gold nanoparticles used as fiducial markers; b) Orthogonal cross sections extracted from the reconstructed volume $\left(X * Y * Z=9593 * 7133 * 3000 \mathrm{~nm}^{3}\right)$. The short white line indicates the position of the slices; b) 3D model of the region indicated by the dashed square in the $X Y$ slice (volume: $X * Y * Z=2400 * 2325 * 1395 \mathrm{~nm}^{3}$ ). The SBAPMMA particles and the surfactant are represented in blue and green, respectively.

As far as the spatial resolution is concerned, the direct estimation $(25 \mathrm{~nm})$ is in very good agreement with the estimations provided by the Crowther's criterion $(23 \mathrm{~nm})$ and the FSC analysis $(26 \mathrm{~nm}$ using 
the $\sigma$-criterion). However, it should be pointed out that the reaching such spatial resolution implies to minimize beam broadening within the water layer (de Jonge, 2018). This can be achieved either by working with very thin but then probably unstable water films, or to consider only objects close to the water upper surface.

\section{Conclusions}

Liquid phase electron microscopy is of growing interest in biology and materials science as it enables the analysis of native objects with (almost) no preparation such as drying or staining. As it provides twodimensional images, it is sometimes difficult to extract the desired structural information. Electron tomography would be a valuable answer. The aim of this study was to demonstrate the feasibility of electron tomography on native liquid suspensions using ESEM. We used an aqueous suspension of latex particles as a model sample, mainly because individual latex particles can be observed only if the suspension has not undergone water evaporation. Indeed, latex particles form homogeneous films upon drying and this transformation is irreversible. The drawback is that polymers give low contrasts in electron microscopy and are highly electron sensitive.

We first optimized and calibrated the rotating stage. Using a procedure well adapted for the acquisition of images with a limited electron dose on the region of interest, we showed that it was possible to acquire tilted image series with both a good stability of the water film and a low level or irradiation damage. Despite artifacts introduced by the presence of a missing wedge and the choice of a large tilt step, the volumes could be reconstructed with standard algorithms and the latex particles could be segmented. It was shown that the latex particles in liquid were assembled in cubic and hexagonal domains. Compared with the cryo SEM, the domains formed by the sample in liquid are smaller than the domains formed by the frozen sample. This was attributed to the influence of the meniscus. Moreover, the comparison between quantitative data extracted from the 3D model and previously published measurements performed on 2D images showed a very good agreement. However, artifacts remained as some latex particles could not be efficiently segmented.

Furthermore, we estimated the spatial resolution in the reconstructed volume in different ways. We used the Crowther's criterion, which assumes a spherical object, and Fourier Shell Correlation, which estimates the resolution from the comparison of two volumes, reconstructed with either odd or even images. We also measured directly the spatial resolution by analyzing a suspension containing latex particles and a surfactant. As the shell formed by the surfactant around the latex particles could be resolved in the 
reconstructed volume, we could reasonably state that the spatial resolution is equal or better than the thickness of the surfactant shell. Interestingly, the value obtained was in very good agreement with the estimations provided by the Crowther's criterion and by Fourier Shell Correlation when taking into account the uncertainty on the measurement (i.e. the pixel size). The resolution calculated at around 25 $\mathrm{nm}$ (3-4 pixels) allowed us to show that the distances between the latex particles could be reduced to the thickness of a surfactant layer. In the future, the spatial resolution could be further reduced in the case of suspensions containing less sensitive materials or objects giving rise to high contrasts.

The experiments described in this work open a new perspective of 3D analysis of samples dispersed in liquid, even beam sensitive or low contrast ones, using STEM at a low electron voltage.

\section{Acknowledgements}

The authors acknowledge the Consortium Lyon Saint-Etienne de Microscopie (CLYM) and the Centre Technologique des Microstructures $(\mathrm{CT} \mu)$ for the access to the microscopes. The authors are grateful to Xavier Jaurand and Sylvie Descartes for their precious help during the cryo-SEM experiments. They also acknowledge the China Scholarship Council (CSC) and the Institut Universitaire de France (IUF) for financial support, as well as BASF for having kindly provided the samples.

\section{References}

Abellan P., Woehl T.J., Parent L.R., Browning N.D., Evans J.E., Arslan I. "Factors influencing quantitative liquid (scanning) transmission electron microscopy", Chem. Commun. 50 (2014), 48734880. DOI: $10.1039 / \mathrm{c} 3 \mathrm{cc} 48479 \mathrm{c}$

Bogner A., Thollet, G., Basset, D., Jouneau, P.H., Gauthier, C. "Wet STEM: a new development in environmental SEM for imaging nano-objects included in a liquid phase”, Ultramicrosc. 104 (2005), 290-301. DOI: 10.1016/j.ultramic.2005.05.005

Chevalier Y., Pichot C., Graillat C., Joanicot M., Wong K., Maquet J., Lindner P., Cabane B. "Film formation with latex particles" Colloid \& Polymer Sci. 270 (1992), 806-821. DOI:

10.1007/BF00776153

Chon C.H., Kihm K.D., Lee S.P., Choi U.S., "Empirical correlation finding the role of temperature and 
particle size for nanofluid $\left(\mathrm{Al}_{2} \mathrm{O}_{3}\right)$ thermal conductivity enhancement", Appl. Phys. Lett. 87 (2005), 153107. DOI: $10.1063 / 1.2093936$

Crowther R.A., Derosier D.J., Klug A., "The reconstruction of a three-dimensional structure from projections and its application to electron microscopy”, Proc. R. Soc. A 317 (1970), 319-340. DOI: 10.1098/rspa.1970.0119

De Jonge N., "Theory of the spatial resolution of (scanning) transmission electron microscopy in liquid water or ice layers", Ultramicrosc. 187 (2018), 113-125. DOI: 10.1016/j.ultramic.2018.01.007

Du Chesne A., Gerharz B., Lieser G., "The segregation of surfactant upon film formation of latex dispersions : an investigation by energy filtering transmission electron microscopy”, Polym. Int. 43 (1997), 187-196. DOI: 10.1002/(SICI)1097-0126(199706)43:2<187::AID-PI752>3.0.CO;2-H

Donald A.M., "The use of environmental scanning electron microscopy for imaging wet and insulating materials". Nature Materials 2 (2003), 511-516. DOI: 10.1038/nmat898

Fedorov A., Beichel R., Kalpathy-Cramer J., Finet J., Fillion-Robin J.C., Pujol S., Bauer C., Jennings D., Fennessy F., Sonka M., Buatti J., Aylward S., Miller J.V., Pieper S., Kikinis R., “3D slicer as an image computing platform for the quantitative imaging network", Magnetic Resonance Imaging 30 (2012), 1323-1341. DOI: 10.1016/j.mri.2012.05.001

Fletcher A.L., Thiel B.L., Donald A.M., "Amplification measurements of alternative imaging gases in environmental SEM". J. Phys. D: Appl. Phys. 30 (1997), 2249-2257. DOI: 10.1088/0022$3727 / 30 / 15 / 018$

Harauz G., Van Heel M., "Exact filters for general geometry three dimensional reconstruction”, Optik 73 (1986), 146-156.

Jan S.P., Choi U.S., "Role of Brownian motion in the enhanced thermal conductivity of nanofluids", Appl. Phys. Lett. 84 (2004), 4316-4318. DOI: 10.1063/1.1756684 
Jornsanoh P., Thollet G., Ferreira J., Masenelli-Varlot K., Gauthier C., Bogner A. "Electron tomography combining ESEM and STEM: a new 3D imaging mode”, Ultramicrosc. 111 (2011), 1247-1254. DOI: 10.1016/j.ultramic.2011.01.041

Kremer J.R., Mastronarde D.N., McIntosh J.R., "Computer visualization of three-dimensional image data using IMOD”, J. Struc. Biol. 116 (1996), 71-76. DOI: 10.1006/jsbi.1996.0013

Masenelli-Varlot K., Malchère A., Ferreira J., Heidari Mezerji H., Bals S., Messaoudi C., Marco Garrido S. "Wet-STEM tomography : principles, potentialities and limitations” Microsc. Microanal. 20 (2014), 366-375. DOI: 10.1017/S1431927614000105

Messaoudi C., Boudier T., Sanchez Sorzano C.O., Marco S., "TomoJ : tomography software for threedimensional reconstruction in transmission electron microscopy”, BMC Bioinformatics 8 (2007), 288. DOI: $10.1186 / 1471-2105-8-288$

Park J., Elmlund H., Ercius P., Yuk J.M., Limmer D.T., Chen Q., Kim K., Han S.H., Weitz D.A., Zettl A., Alivisatos A.P., "3D structure of individual nanocrystals in solution by electron microscopy”, Science 349 (2015), 290-295. DOI: 10.1126/science.aab1343

Peckys D.B., Baudoin J.P., Eder M., Werner U., de Jonge N. "Epidermal growth factor receptor subunit locations determined in hydrated cells with environmental scanning electron microscopy”, Sci. Rep. 3 (2013), 2626. DOI: 10.10138/srep02626

Perret A., Foray G., Masenelli-Varlot K., Maire E., Yrieix B., "Study of the surfactant role in latexaerogel systems by scanning transmission electron microscopy on aqueous suspensions", J. Microsc. 269 (2018), 3-13. DOI: 10.1111/jmi.12603

Proetto M.T., Rush A.M., Chien M.P., Abellan Baeza P., Patterson J.P., Thompson M.P., Olson N.H., Moore C.E., Rheingold A.L., Andolina C., Millstone J., Howell S.B., Browning N.D., Evans J.E., Gianneschi N.C., "Dynamics of soft nanomaterials captured by transmission electron lmicroscopy in 
liquid water", J. Am. Chem. Soc. 136 (2014), 1162-1165. DOI:10.1021/ja408513m.

Roiban L., Foray G., Rong Q., Perret A., Ihiawakrim D., Masenelli-Varlot K., Maire E., Yrieix B., "Advanced three dimensional characterization of silica-based ultraporous materials", RSC Advances 13 (2016), 10625-10632. DOI: 10.1039/C5RA26014K

Ross F.M., “Opportunities and challenges in liquid cell electron microscopy”, Science 350 (2015), aaa9886. DOI: 10.116/science.aaa9886

Schneider N.M., Norton M.M., Mendel B.J., Grogan J.M., Ross F.M., Bau H.H. “Electron-water interactions and implications for liquid cell electron microscopy", J. Phys. Chem. C 118 (2014), 2237322382. DOI: 10.1021/jp507400n

Stokes, D.J. "Principles and practive of variable pressure environmental scanning electron microscopy (VP-ESEM)", Wiley-Blackwell, RMS, 2008. ISBN: 978-0-470-06540-2

Tan J.P.Y., Tan H.R., Boothroyd C., Foo Y.L., He C.B., Lin, M., "Three dimensional structure of $\mathrm{CeO}_{2}$ nanocrystals”, J. Phys. Chem. C 115 (2011), 3544-3551. DOI: 10.1021/jp1122097

Tzitzinou A., Jenneson P.M., Clough A.S., Keddie J.L., Lu J.R., Zhdan P., Treacher K.E., Satguru R., "Surfactant concentration and morphology at the surfaces of acrylic latex films", Progress in Organic Coatings 35 (1999), 89-99. DOI: 10.1016/S0300-9440(99)00017-X

Van Heel M., Schatz M., "Fourier shell correlation threshold criteria”, J. Struct. Biol. 151 (2005), 250262. DOI: $10.1016 /$ j.jsb.2005.05.009

Verch A., Pfaff M., de Jonge N., "Exceptionally slow movement of gold nanoparticles at a solid/liquid interface investigated by scanning transmission electron microscopy”, Langmuir 31 (2015), 6956-6964. DOI: 10.1021/acs.langmuir.5b00150

Xiao J., Foray G., Masenelli-Varlot K., “Analysis of liquid suspensions using scanning electron 
microscopy in transmission: estimation of the water film thickness using Monte Carlo simulations", J. Microsc. 269 (2018), 151-160. DOI: 10.1111/jmi.12619

Yuk J.M., Park J., Ercius P., Kim K., Hellebush D.J., Crommie M.F., Lee J.Y., Zettl A., Alivisatos A.P., "High-resolution EM of colloidal nanocrystal growth using graphene liquid cells", Science 336 (2012), 61. DOI: $10.1126 /$ science. 1217654

Ziegler C., Eychmüller A., "Seeded Growth Synthesis of Uniform Gold Nanoparticles with Diameters of 15-300 nm”, J. Phys. Chem. C 115, (2011), 4502-4506. DOI: 10.1021/jp1106982 